\title{
Necrotizing Soft Tissue Infections: Our Experience at Govt. Royapettah Hospital.
}

\author{
Dr. S. Thirunavukkarasu. ${ }^{1}$, Dr.P. Ragumani ${ }^{2}$ \\ ${ }^{1}$ (Department Of General Surgery, Madras medical College, Chennai, India) \\ ${ }^{2}$ (Department Of General Surgery, .Madras Medical College, Chennai, India)
}

\begin{abstract}
:
Background: Necrotizing soft tissue infections (NSTIs) are a group of highly morbid and lethal infections. Successful management entails early recognition, aggressive operative debridement, aggressive appropriate antibiotics and supportive care.

Aims: to find out the age \& gender distribution, category and etiological factors of NSTIS

ii) To identify the prognostic factors and mortality rate of the same.

Materials and Methods: A descriptive study of 42 patients, who were admitted with NSTIs at Govt. Royapettah hospital were done between January 2015 and December 2015.

Results: Most common category of NSTI was found to be Necrotizing fasciitis with male preponderance, with a mean age of 49 years. Significant association was found between NSTIs and Diabetes \& Anemia. Idiopathic etiology (45\%) was most common in our study followed by trauma(43\%).

Conclusions: Necrotizing soft tissue infections are fulminant polymicrobial soft tissue infections with a high mortality rate. Early diagnosis of the presence of a NSTI is critical if optimal outcomes are to be achieved

Keywords: NSTIs - Necrotizing soft tissue infection, Necrotizing fasciitis, Fournier's gangrene.

\section{Introduction}

Necrotizing soft tissue infection ( NSTI) is the current term used to describe severe soft tissue infection with associated tissue necrosis regardless of anatomic region or depth of tissue involvement. This term encompasses previously described multiple categories of soft tissue infections including necrotizing cellulitis, necrotizing fasciitis, myositis, myonecrosis, Fournier's gangrene and gas gangrene. Necrotizing soft tissue infections continue to have high mortality and post enormous diagnostic and therapeutic challenges.For optimal outcome, treatment includes appropriate antibiotic coverage and early wide local surgical excision. Recovery requires aggressive resuscitation, post-operative nutritional support and wound care similar to that of burn patients in many aspects.
\end{abstract}

\section{Materials And Methods}

A descriptive study of 42 patients who were admitted with NSTIs at Govt. Royapettah hospital were done between January 2015 to December 2015. INCLUSION CRITERIA: All the Necrotizing soft tissue infection patients included. EXCLUSION CRITERIA: children <12, Diabetic foot syndrome and limb ischemia due to vascular occulusion. The patients were evaluated with baseline investigations, pus culture \& sensitivity and appropriate treatment in the form of surgical debridement and antibiotics initially broad spectrum antibiotics started and then appropriate antibiotics were given after seeing the culture results. The age, gender, presenting complaints , anatomical site , microbiological isolates and co-morbid conditions like CKD, Diabetes, Ischemic heart disease were documented by a common proforma. The results were tabulated and analysed. Stastical significance was calculated using software SPSS ,version 20.0 and compared.

\section{Results}

Necrotizing soft tissue infections were commonly seen in men . The male : female ratio being 5:1. We find that the prevalence of necrotizing soft tissue infections were highest among age group of 61- 80 years , 13( male : $13 \&$ female : 0 ) followed by age of 31 to 40 years, 11 ( male : 8 , female : 3).Interestingly we also found 3 cases of necrotising soft tissue infection found between age group $21-30$.Mean age of the disease is 49 years. Most common presenting symptom was pain in the local region followed by skin slough/ necrosis and swelling. Fever was not very common accounting for less than 50\% of affected individuals. We found that predominant NSTIs werePolymicrobial( Type 1) 60\% and Monomicrobial ( Type 2) 40\%. Type 3 infections were absent in our study .Polymicrobial infections showed a predominance of E.coli in culture followed by Klebsiella and Streptococci. Monomicrobial infections were isolatesof Staphylococci followed by Streptococci. The predominant infection subtype is Necrotising Fascitis accounting for 83\% of NSTIs. Followed by Fournier's gangrene (10\%). Lower extremities were the most common site of occurrence of NSTIs (64\%), 
followed by upper extremities ( $17 \%$ ) and scrotum (12\%). Most common etiological factor amongst established causes was found to be inciting trauma associated with 18 ( 14 blunt and 4 penetrating injury) out of 42 patients accounting for $43 \%$. 19 patients (45\%) did not have any established etiological factor( idiopathic). We encountered 2 post-operative patients developing NSTIs , one being a case of DU perforation / Laparotomy done developed scrotal necrotizing cellulitis on the third postoperative day and a case of perianal abcess / Incision\& Drainage done developed Abdominal wall necrotizing fasciitis postoperatively. As a primary surgery ,16 patients required wound debridement (slough excision) alone on presentation and 26 patients needed additional decompressive fasciotomy with wound debridement. Further after regular wound dressings, antibiotics and other general supportive measures 22 patients required Split Skin Graft as a form of secondary surgery for complete healing of wound. $36(86 \%)$ Patients needed less than 2 debridements and only 6 patients required 3 or more debridements.All debridements were done under anaesthesia . 40 patients recovered completely and 2 expired. Time of presentation to hospital and timing of the earliest debridement proved to be the most important factor to survival.

$30(71 \%)$ patients had haemoglobin levels less than $<12 \mathrm{~g} \%$. WBC elevation of $>15000 / \mathrm{cu} . \mathrm{mm}$ was seen in 19 patients( 45\%). Elevated renal parameters were seen in 7 patients out of which 3 had pre-existing CKD . Diabetes was the most common co-morbid condition associated with NSTIs accounting for 22 patients $(52 \%)$. Mortality was seen in 2 patients $(4.7 \%)$. Both were male. The factors associated with mortality included old age > 60 years, delayed presentation to hospital with sepsis, co-morbid illness like Diabetes, Systemic Hypertension, IHD and CKD.Both patients had received early resuscitation and adequate debridement, but because of the forementioned factors we lost the patients in less than 48 hours of hospital stay. The mean hospital stay for survivors ranged from minimum of 18 days to 90 days for complete recovery with an average of 40 days. Favourable factors for early recovery included young age, traumatic etiology and no associated comorbid illness. Female patients had overall better recovery compared to their male counterparts. All the results of the study except Microbiological isolates were found to be statistically significant with Level 1 significance ( $\mathrm{p}<0.01)$.

\section{Discussion}

Necrotizing soft tissue infections (NSTIs) are fulminant infections of any layer of the soft tissue compartment associated with widespread necrosis, systemic toxicity and a high mortality rate if not treated early. These infections were first described by Jones in 1871 and at that time they were termed"hospital gangrene". The incidence of NSTIs in the United States of America is estimated to be around 500-1500 cases per year. The mortality rates have been reported to be around $25 \%$, although it is higher in some studies.

Based on the microbiological characteristics, NSTIs are

broadly classified into three types.

Type I Infections: Type I infections are the most common forms accounting for $80 \%$ or more of all the NSTIs, Fournier's gangrene etc. These are mostly polymicrobial infections having a combination of aerobes, anaerobes and facultative aerobes/anaerobes.The common aerobic species isolated from these infections are streptococci, staphylococci, enterococci, and the family of gram-negative rods. Bacteroidesspecies are the most common anaerobes involved.

Type II Infections: Type II infections are usually monomicrobial and usually follow a minor injury accounting for $10-15 \%$ of all NSTIs . Common organisms involved are the Group A beta-hemolytic streptococcus or the $S$. aureus.

Type III Infections: These account for less than5\% of all NSTIs and clostridial gas forming myonecrosis is the prototype of type III infections, most commonly caused by $\mathrm{Cl}$. Perfringens . Necrotizing soft tissue infections are also caused by Vibrio vulnificusand Aeromonashydrophilaand various fungi as Mucor, Rhizopus, or Rhizomucor.

\section{Investigations and Management:}

Along with routine investigations, pus culture \& sensitivity, imaging radiography of local part are commonly done. CT, MRI and Histopathology are done when diagnosis is in doubt and appropriately. Management involves adequate surgical debridement, initially broad spectrum antibiotics like piperacillin and tazobactum, cefoperazone and sulbactum and then sensitive antibiotics administration, daily dressing , supportive care in the form of nutrition.

\section{Comparison with our results:}

- Mean age of presentation in our study was found to be 49 years which was comparable to 53 years by Yana et al 2015 . 
- Males were commonly affected ratio being 5:1comparable to the previous mentioned study of NSTIs by Yana et al and supported by literature

- Extremities was the most common site of occurrence of NSTIs in our study accounting for $81 \%$ comparable to Review article of NSTIs by Daniel Anaya et al 2015.

- Etiology : Most common etiology in our study was idiopathic $45 \%$ followed by trauma $43 \%$. Review article by John et al 2015 and literature quote trauma to be the most common precipitating factor.

\begin{tabular}{|l|l|l|}
\hline \multicolumn{3}{|c|}{ 2) Age distribution: } \\
\hline AGE & Male & Female \\
\hline $21-30$ & 3 & -- \\
\hline $31-40$ & 8 & 3 \\
\hline $41-50$ & 5 & 2 \\
\hline $51-60$ & 6 & 2 \\
\hline $61-70$ & 9 & -- \\
\hline $71-80$ & 4 & -- \\
\hline
\end{tabular}

- Co-morbid conditions: Diabetes was consistently the most common associated co-morbid condition associated with NSTIs supported by all literatures.

- Microbiological isolates were polymicrobial in $60 \%$ and $40 \%$ monomicrobial. E.coli predominance was seen in polymicrobial and Staphylococci in monomicrobial . But results were insignificant ( pvalue0.113).

- Mortality rates:Our study shows mortality of $4.7 \%$ found to lesser compared to standard rates of $25 \%$ ( Yana-pucket et al 2015), 23.5\% ( Camile Hua et al 2015). Literature documents a mortality of upto $35 \%$

\section{V) Figures And Tables :}

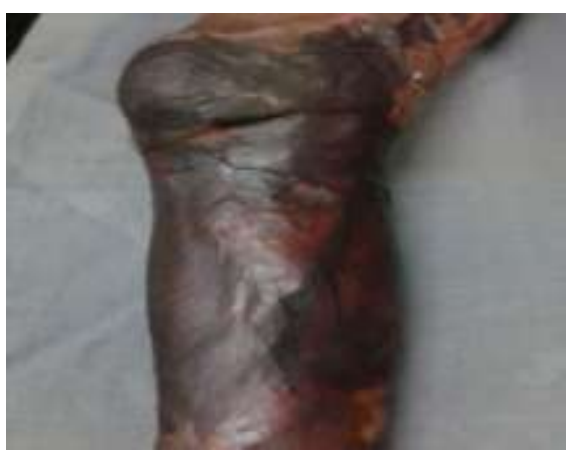

NECROTIZING FASCITIS

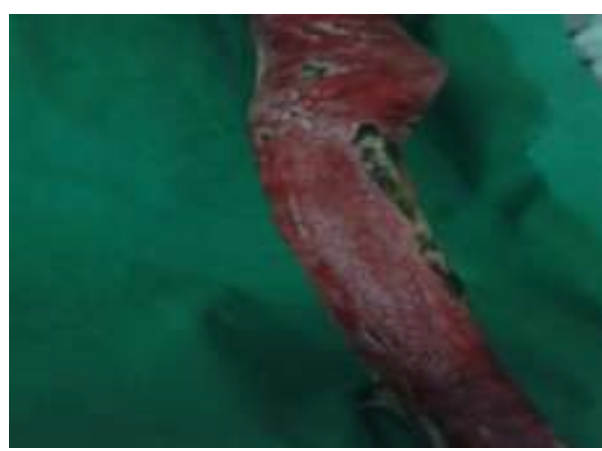

POST WOUND DEBRIDEMENT

\begin{tabular}{|l|l|}
\hline \multicolumn{2}{|c|}{ 1)Sex distribution: } \\
\hline \multicolumn{2}{|c|}{ 1)Sex Distribution } \\
\hline Male & Female \\
\hline 35 & 7 \\
\hline
\end{tabular}

\begin{tabular}{|l|l|l|}
\hline \multicolumn{2}{|c|}{ 3) Organism Isolates } \\
\hline Micro-organism & $\begin{array}{l}\text { Poly } \\
\text { microbial }\end{array}$ & Monomicrobial \\
\hline Streptococcus species & 4 & 3 \\
\hline Staphlococcus species & 6 & 9 \\
\hline Klebsiella & 7 & 1 \\
\hline Eschericia coli & 11 & -- \\
\hline Pseudomonas aeroginosa & 8 & 3 \\
\hline Proteus & 6 & -- \\
\hline Enterobacter & 4 & -- \\
\hline Bacteroides & 3 & -- \\
\hline MRSA & 1 & -- \\
\hline Enterococcus & -- & 1 \\
\hline
\end{tabular}

\begin{tabular}{|l|l|}
\hline \multicolumn{2}{|c|}{ 4) Category of Soft tissue infection: } \\
\hline Type of infection & No of patients \\
\hline Necrotising Fascitis & 35 \\
\hline Necrotising Cellulitis & 2 \\
\hline Myonecrosis & 1 \\
\hline Fournier's Gangrene & 4 \\
\hline
\end{tabular}




\begin{tabular}{|l|l|}
\hline \multicolumn{2}{|c|}{ 5) Site: } \\
\hline Site & No of patients \\
\hline Scrotum & 5 \\
\hline Upper extremity & 7 \\
\hline Lower extremity & 27 \\
\hline Gluteal region & 1 \\
\hline Trunk & 2 \\
\hline
\end{tabular}

\begin{tabular}{|l|l|}
\hline \multicolumn{2}{|c|}{ 6)Precipitating Factors: } \\
\hline Cause: & No of patients: \\
\hline Blunt Trauma & 14 \\
\hline Thorn prick & 1 \\
\hline Bangle Prick & 1 \\
\hline Intermuscular Injection & 2 \\
\hline Human bite & 1 \\
\hline Dog bite & 1 \\
\hline Immunosuppression / Steroid intake & 1 \\
\hline Post operative & 2 \\
\hline Idiopathic & 19 \\
\hline
\end{tabular}

\begin{tabular}{|l|l|l|}
\hline \multicolumn{2}{|c|}{ 7) Presenting Complaints } \\
\hline SYMPTOMS & $\begin{array}{l}\text { No of Patients - } \\
\text { PRESENT }\end{array}$ & $\begin{array}{l}\text { No of Patients } \\
\text {-ABSENT }\end{array}$ \\
\hline Pain & 36 & 6 \\
\hline Oedema / Swelling & 31 & 11 \\
\hline Discolouration & 10 & 32 \\
\hline Fever & 17 & 25 \\
\hline Vesicles & 5 & 37 \\
\hline Discharge & 19 & 23 \\
\hline Crepitus & 6 & 36 \\
\hline Induration & 15 & 27 \\
\hline Skin slough/ necrosis & 32 & 10 \\
\hline
\end{tabular}

\section{SECONDARY SURGERY:}

\begin{tabular}{|l|l|}
\hline AMPUTATION & 5 \\
\hline SPIT SXIN GRAFT & 22 \\
\hline FLAP COVER & 2 \\
\hline SECONDARY SUTURING & 7 \\
\hline
\end{tabular}

\begin{tabular}{|l|l|}
\hline \multicolumn{2}{|c|}{ Lab values: } \\
\hline $\mathrm{Hb}<12 \mathrm{~g} \%$ & 30 \\
\hline \multicolumn{2}{|c|}{ Lab values: } \\
\hline $\mathrm{Hb}<12 \mathrm{~g} \%$ & 30 \\
\hline Total count $>15,000$ & 19 \\
\hline $\begin{array}{l}\text { Elevated Renal parameters } \\
\text { Urea }>40 \text { mg } \\
\text { Creatinine }>1.4 \mathrm{mg}\end{array}$ & 7 \\
\hline Hypoalbuminemia & 3 \\
\hline Blood Sugar $>180 \mathrm{mg} \%$ & 22 \\
\hline $\begin{array}{l}\text { Serum Sodium level <135 } \\
\text { mmol/L }\end{array}$ & 17 \\
\hline
\end{tabular}




\begin{tabular}{|l|c|}
\hline \multicolumn{2}{|c|}{ 8) Surgery: } \\
\hline DEBRIDEMENT & 16 \\
\hline DEBRIDEMENT \& FASCIOTOMY & 26 \\
\hline
\end{tabular}

\begin{tabular}{|l|l|}
\hline \multicolumn{2}{|c|}{ 9) No of debridements: } \\
\hline No of debridements & Patients \\
\hline 1 & 19 \\
\hline 2 & 17 \\
\hline 3 and more & 6 \\
\hline
\end{tabular}

\section{Conclusion}

Necrotizing soft tissue infections are fulminant polymicrobial soft tissue infections with a high mortality rate. The treatment of all of these variants depends on prompt diagnosis, ideally based on physical examination, resuscitation of the patient, meticulous surgical removal of the nonviable tissue, appropriate and timely IV antibiotic therapy, and usually second-look surgery. While the mortality from these infections has dropped significantly in the last century, under treated lesions still remain a source of mortality, the most common error in the management of these lesions is delayed or inadequate surgical debridement. Early diagnosis with a high index of clinical suspicion would definitely reduced both morbidity and mortality.Timing of intervention plays a key role in overall survival and prognosis.

\section{References}

[1]. Necrotising Soft tissue infections article by Daniel Anaya et al 2015

[2]. Clinical comparison of fournier's gangrene to other necrotizing soft tissue infections (Yana Puckett, Brian Fisher and SharmilaDissanaike 2015)

[3]. Prognostic factors in necrotizing soft-tissue infections (NSTI): A cohort study ( Camile Hua et al 2015 )

[4]. Postgrad Med J 1999;75:645-649 (C) The Fellowship of Postgraduate Medicine, 1999 John et al Hakkarainen TW, Kopari NM, Pham TN and Evans HL. Necrotizing soft tissue infections: review and current concepts in treatment, systems of care, and outcomes. CurrProbl Surg. 2014;51:344-62.

[5]. Fabri PJ. Necrotizing soft tissue infections. J Am Coll Surg. 2014; 218:302-3

[6]. Kaafarani HM and King DR. Necrotizing skin and soft tissue infections. SurgClin North Am. 2014; 94:155-63.

[7]. Hussein QA and Anaya DA. Necrotizing soft tissue infections. Crit Care Clin. 2013; 29:795-806.

[8]. Shiroff AM, Herlitz GN and Gracias VH. Necrotizing soft tissue infections. J Intensive Care Med. 2014; 29:138-44.

[9]. Mullangi PK and Khardori NM. Necrotizing soft-tissue infections. Med Clin North Am. 2012;96:1193- 202. 